\title{
Molecular profiling of advanced solid tumors and patient outcomes with genotype-matched clinical trials: the Princess Margaret IMPACT/COMPACT trial
}

Tracy L. Stockley ${ }^{1,2,3}$, Amit M. Oza ${ }^{4,5}$, Hal K. Berman ${ }^{1,2}$, Natasha B. Leigh| ${ }^{4,5}$, Jennifer J. Knox ${ }^{4,5}$, Frances A. Shepherd ${ }^{4,5}$, Eric X. Chen ${ }^{4,5}$, Monika K. Krzyzanowska ${ }^{4,5}$, Neesha Dhani ${ }^{4,5}$, Anthony M. Joshua ${ }^{4,5}$, Ming-Sound Tsao ${ }^{1,2,6}$, Stefano Serra ${ }^{1,2}$, Blaise Clarke ${ }^{1,2}$, Michael H. Roehrl ${ }^{1,2,6}$, Tong Zhang ${ }^{1}$, Mahadeo A. Sukhai ${ }^{1}$, Nadia Califaretti ${ }^{7,8}$, Mateya Trinkaus ${ }^{9}$, Patricia Shaw ${ }^{1,2}$, Theodorus van der Kwast ${ }^{1,2}$, Lisa Wang ${ }^{10}$, Carl Virtanen ${ }^{3,11}$, Raymond H. Kim ${ }^{3,4,5}$, Albiruni R. A. Razak ${ }^{4,5}$, Aaron R. Hansen ${ }^{4,5}$, Celeste Yu ${ }^{3}$, Trevor J. Pugh 3,6,11, Suzanne Kamel-Reid ${ }^{1,2,3,6}$, Lillian L. Siu ${ }^{3,4,5}$ and Philippe L. Bedard ${ }^{3,4,5^{*}}$

\begin{abstract}
Background: The clinical utility of molecular profiling of tumor tissue to guide treatment of patients with advanced solid tumors is unknown. Our objectives were to evaluate the frequency of genomic alterations, clinical "actionability" of somatic variants, enrollment in mutation-targeted or other clinical trials, and outcome of molecular profiling for advanced solid tumor patients at the Princess Margaret Cancer Centre (PM).

Methods: Patients with advanced solid tumors aged $\geq 18$ years, good performance status, and archival tumor tissue available were prospectively consented. DNA from archival formalin-fixed paraffin-embedded tumor tissue was tested using a MALDI-TOF MS hotspot panel or a targeted next generation sequencing (NGS) panel. Somatic variants were classified according to clinical actionability and an annotated report included in the electronic medical record. Oncologists were provided with summary tables of their patients' molecular profiling results and available mutationspecific clinical trials. Enrolment in genotype-matched versus genotype-unmatched clinical trials following release of profiling results and response by RECIST v1.1 criteria were evaluated.

Results: From March 2012 to July 2014, 1893 patients were enrolled and 1640 tested. After a median follow-up of 18 months, 245 patients (15\%) who were tested were subsequently treated on 277 therapeutic clinical trials, including 84 patients (5\%) on 89 genotype-matched trials. The overall response rate was higher in patients treated on genotypematched trials (19\%) compared with genotype-unmatched trials (9\%; $p<0.026)$. In a multi-variable model, trial matching by genotype $(p=0.021)$ and female gender $(p=0.034)$ were the only factors associated with increased likelihood of treatment response.

(Continued on next page)
\end{abstract}

* Correspondence: philippe.bedard@uhn.ca

${ }^{3}$ Cancer Genomics Program, Princess Margaret Cancer Centre, Toronto,

Canada

${ }^{4}$ Division of Medical Oncology and Hematology, Princess Margaret Cancer

Centre, 610 University Avenue, Toronto M5G 2M9, Canada

Full list of author information is available at the end of the article

(c) The Author(s). 2016 Open Access This article is distributed under the terms of the Creative Commons Attribution 4.0 International License (http://creativecommons.org/licenses/by/4.0/), which permits unrestricted use, distribution, and reproduction in any medium, provided you give appropriate credit to the original author(s) and the source, provide a link to the Creative Commons license, and indicate if changes were made. The Creative Commons Public Domain Dedication waiver (http://creativecommons.org/publicdomain/zero/1.0/) applies to the data made available in this article, unless otherwise stated. 
(Continued from previous page)

Conclusions: Few advanced solid tumor patients enrolled in a prospective institutional molecular profiling trial were treated subsequently on genotype-matched therapeutic trials. In this non-randomized comparison, genotypeenrichment of early phase clinical trials was associated with an increased objective tumor response rate.

Trial registration: NCT01505400 (date of registration 4 January 2012).

Keywords: Molecular profiling, DNA sequencing, Clinical trials, Solid tumors, Precision medicine

\section{Background}

Molecular profiling can provide diagnostic, prognostic, or treatment-related information to guide cancer patient management. Advances in next-generation sequencing (NGS) have enabled multiplex testing to overcome the constraints associated with sequential single-analyte testing [1-3]. Large-scale research projects have elucidated genomic landscapes of many cancers but have provided limited insight into the clinical utility of genomic testing. Our aim was to evaluate if targeted DNA profiling improves outcomes for patients assigned to clinical trials based on knowledge of actionable somatic mutations.

At the Princess Margaret Cancer Centre (PM), the Integrated Molecular Profiling in Advanced Cancers Trial (IMPACT) and Community Molecular Profiling in Advanced Cancers Trial (COMPACT) are prospective studies that provide molecular characterization data to oncologists to match patients with advanced solid tumors to clinical trials with targeted therapies. Here, we report the frequency of alterations, clinical "actionability" of the somatic variants, clinical trial enrollment, and outcome based upon molecular profiling results.

\section{Methods}

\section{Patient cohort}

For IMPACT, patients with advanced solid tumors treated at PM were prospectively consented for molecular profiling during a routine clinical visit. For COMPACT, patients with advanced solid tumors treated at other hospitals in Ontario were referred to a dedicated weekly clinic at PM for eligibility review, consent, and blood sample collection. Eligible patients had advanced solid tumors, were aged $\geq 18$ years, had Eastern Cooperative Oncology Group (ECOG) performance status $\leq 1$, and had available formalin-fixed paraffin-embedded (FFPE) archival tumor tissue. The University Health Network Research Ethics Board approved this study (\#11-0962-CE). Enrollment for IMPACT began on 1 March 2012 and for COMPACT on 16 November 2012 and ended on 31 July 2014 for this analysis.

\section{Specimens}

DNA was extracted from sections of FFPE tumor specimens from biopsies or surgical resections. If multiple archival tumor specimens were available, the most recent archival FFPE specimen was reviewed, with a minimum acceptable tumor cellularity of $10 \%$. Tumor regions were isolated by 1-2 $\times 1 \mathrm{~mm}$ punch from FFPE blocks or manual macrodissection of unstained material from 15-20 slides. FFPE samples were deparaffinized, cells lysed with proteinase $\mathrm{K}$, and DNA extracted using the QIAmp DNA FFPE Tissue Kit (Qiagen, Germantown, MD, USA). DNA was quantified using the Qubit dsDNA Assay kit on the Qubit 2.0 Fluorometer (ThermoFisher Scientific, Waltham, MA, USA).

Participants provided a peripheral blood sample $(5 \mathrm{~mL}$ in EDTA-coated tubes) as a source of matched germline DNA. DNA was extracted using either standard manual phenol/chloroform extraction methods or automated extraction (MagAttract DNA Mini M48 kit; Qiagen). Patients were offered return of pathogenic germline results at the time of consent and asked to identify a family member delegate who could receive results on their behalf if required.

\section{Molecular profiling assays}

All testing was performed in a laboratory accredited by the College of American Pathologists (CAP) and certified to meet Clinical Laboratory Improvement Amendments (CLIA). Three molecular profiling assays were used over the study period: a custom multiplex genotyping panel on a matrix-assisted laser desorption/ionization time-of-flight (MALDI-TOF) mass-spectrometry platform (MassARRAY, Agena Bioscience, San Diego, CA, USA) to genotype 279 mutations within 23 genes (Additional file 1: Table S1); the TruSeq Amplicon Cancer Panel (TSACP, Illumina) on the MiSeq sequencer (Illumina) covering regions of 48 genes (Additional file 1: Table S2); and the Ion AmpliSeq Cancer Panel (ASCP, ThermoFisher Scientific) on the Ion Proton sequencer (ThermoFisher Scientific) covering regions of 50 genes (Additional file 1: Table S3). For more in-depth methodology on molecular profiling assays, including sequence alignment and base calling, see Additional file 1: Supplementary Methods.

\section{Variant assessment and classification}

Variants were assessed and classified according to the classification scheme of Sukhai et al. [4]. Briefly, a fiveclass scheme was used to sort variants according to actionability (defined as providing information on prognosis, 
prediction, diagnosis, or treatment), recurrence of variants in specific tumor sites, and known or predicted deleterious effects on protein function. Interpretation and data integration were performed using Alamut v.2.4.5 (Interactive Biosoftware, Rouen, France). Primary review, assessment, and classification of all variants were independently performed by a minimum of two assessors followed by a third review prior to reporting, with cases where assessors disagreed resolved by group discussion.

\section{Immunohistochemistry (IHC)}

Phosphatase and tensin homolog (PTEN) IHC was performed using rabbit monoclonal Ab 138G6 (Cell Signaling Technology, Danvers, MA, USA) on a Dako platform using a dilution of 1:50 and Flex +30 protocol. Complete absence of tumor cell staining with positive staining of surrounding tumor stroma fibroblasts/endothelial cells was used to denote PTEN deficiency [5].

\section{Return of testing results}

The molecular profiling report was included in the electronic medical record and returned to the treating oncologist. The clinical significance of profiling results was discussed with PM patients during a routine clinic visit by their treating oncologist. A PM oncologist reviewed results with patients treated at other hospitals by telephone. All oncologists were provided with regular summary tables of testing results and mutation-specific clinical trial listings available at PM. A monthly genomic tumor board was convened at PM to establish consensus treatment recommendations for patients with complex profiling results. A committee consisting of a molecular geneticist, medical geneticist, genetic councilor, and medical oncologist reviewed pathogenic germline variants before return of germline testing results. Germline results were disclosed to the patient or designate by a genetic counselor or medical geneticist.

\section{Clinical data collection}

For each patient, baseline patient and tumor characteristics, treatment regimen(s), time on treatment(s) and survival were retrieved from medical records and updated every three months. Therapeutic clinical trial enrollment was evaluated from the date of reporting molecular profiling results until 9 January 2015. Genotype-matched trials were defined as studies with eligibility criteria restricted to patients with specific somatic mutations, those with a targeted drug with enriched clinical or preclinical activity in a patient's genotype, or those with a drug that inhibited a pathway directly linked to the somatic mutation. Decisions about trial enrollment were based upon trial availability, patient or physician preference, and did not follow a pre-specified algorithm.
Targeted lesion measurements and RECIST 1.1 [6] assessments were performed by radiologists.

\section{Statistics}

Descriptive statistics were used to summarize patient characteristics, profiling results, and anti-tumor activity. Comparisons between patients with profiling results treated on genotype-matched and genotype-unmatched trials were performed using a generalized estimating equation (GEE) model [7]. A multi-variable GEE model for response included trial matching by genotype, gender, trial phase, number of lines of prior systemic therapy, investigational agent class, age, tumor type, and sequencing platform. A mixed model was used to compare time on treatment, defined as the date of trial enrollment until the date of discontinuation of investigational treatment. A robust score test was used to compare overall survival following trial enrolment between genotype-matched and genotype-unmatched groups [8]. These comparisons accounted for individual patients who were included on multiple therapeutic trials [8]. Differences with $p$ values of $<0.05$ were considered statistically significant.

\section{Results}

\section{Patient cohort}

A total of 1893 patients were enrolled, including gynecological (23\%), breast (18\%), lung (18\%), colorectal $(17 \%)$, pancreatobiliary $(8 \%)$, upper aerodigestive (6\%), genitourinary (5\%), and other (5\%) cancers (Table 1 ). The median age was 59 years (age range, $18-89$ years); patients were predominantly female (69\%); had received a median of 2 prior systemic treatments (range, $1-18$ ), and had excellent performance status (43\% PS0 and $56 \%$ PS1). Of 253 (13\%) screen failures, $10 \%$ were for insufficient tissue or DNA and $3 \%$ for clinical deterioration or other reasons. The median follow-up from reporting results was 18 months (range, $1-33$ months). A total of 651 (40\%) patients were deceased at the time of the database lock.

\section{Molecular profiling}

Successful molecular profiling was achieved in 1640 patients (87 \%), 827 (50 \%) had samples tested by MALDI-TOF MS, 792 (48 \%) by TSACP, and 21 (1\%) by ASCP (Fig. 1). One or more somatic mutations were detected in 341 (41 \%) patients tested by MALDI-TOF MS, $583(74 \%)$ by TSACP, and 14 (67 \%) by ASCP. Median laboratory turnaround time (sample receipt to report) was 32 days (range, 6-228 days). Of patient samples tested by MALDI-TOF MS, KRAS (21\%) was the most frequently mutated gene, followed by PIK $3 C A$ (12\%), with additional genes in the range of $1-5 \%$ frequency. Of samples tested by the TSACP, TP53 had the highest mutation frequency ( $47 \%$ of all identified 
Table 1 Characteristics of patients enrolled into IMPACT/COMPACT ( $n=1893$ )

\begin{tabular}{lllll}
\hline Patients enrolled & Patients enrolled & Patients profiled & $\begin{array}{l}\text { Patients profiled enrolled on } \\
\text { any therapeutic trials }\end{array}$ & $\begin{array}{l}\text { Patients profiled enrolled on } \\
\text { genotype-matched trials }\end{array}$ \\
\hline Median age (range) & $59(18-89)$ & $58(18-89)$ & $58(18-81)$ & $58(24-81)$ \\
Female/Male & $1303 / 590(59 \%) /(31 \%)$ & $1166 / 479(71 \%) /(29 \%)$ & $205 / 72(74 \%) /(26 \%)$ & $64 / 25(72 \%) /(28 \%)$ \\
Median lines of prior treatment (range) & $2(1-18)$ & $2(1-18)$ & $2(1-16)$ & $2(1-11)$ \\
ECOG performance status (0/1/2) & $43 \% / 56 \% /<1 \%$ & $44 \% / 55 \% /<1 \%$ & $78 \% / 22 \% / 0 \%$ & $78 \% / 22 \% / 0 \%$ \\
Median time from collection of archival tumor sample to & $1.6(0.1-24.9)$ & $1.7(0.1-18.9)$ & $1.9(0.1-18.9)$ \\
profiling report in years (range) & & $1080 / 560$ & $193 / 84$ & $66 / 23$ \\
Primary lesion/Metastatic lesion profiled & & $(70 \%) /(30 \%)$ & $(74 \%) /(26 \%)$ \\
& & $(66 \%) /(34 \%)$ & $41 / 310(13 \%)$ & $19 / 310(6 \%)$ \\
Tumor types & & $38 / 299(13 \%)$ & $18 / 299(6 \%)$ \\
Breast & $341(18 \%)$ & $299(18 \%)$ & $80 / 405(20 \%)$ & $20 / 405(5 \%)$ \\
Colorectal & $326(17 \%)$ & $405(25 \%)$ & $43 / 256(17 \%)$ & $18 / 256(7 \%)$ \\
Gynecological & $430(23 \%)$ & $256(16 \%)$ & $9 / 74(12 \%)$ & $4 / 74(5 \%)$ \\
Lung & $339(18 \%)$ & $74(5 \%)$ & $9 / 104(9 \%)$ & $1 / 104(1 \%)$ \\
Genitourinary & $104(6 \%)$ & $8 / 102(8 \%)$ & $17 / 81(21 \%)$ & $2 / 102(2 \%)$ \\
Pancreatobiliary & $92(5 \%)$ & $102(6 \%)$ & $245 / 1640(15 \%)$ & $2 / 81(2 \%)$ \\
Upper aerodigestive & $151(8 \%)$ & 1640 & & $84 / 1640(5 \%)$ \\
Other & $115(6 \%)$ & &
\end{tabular}

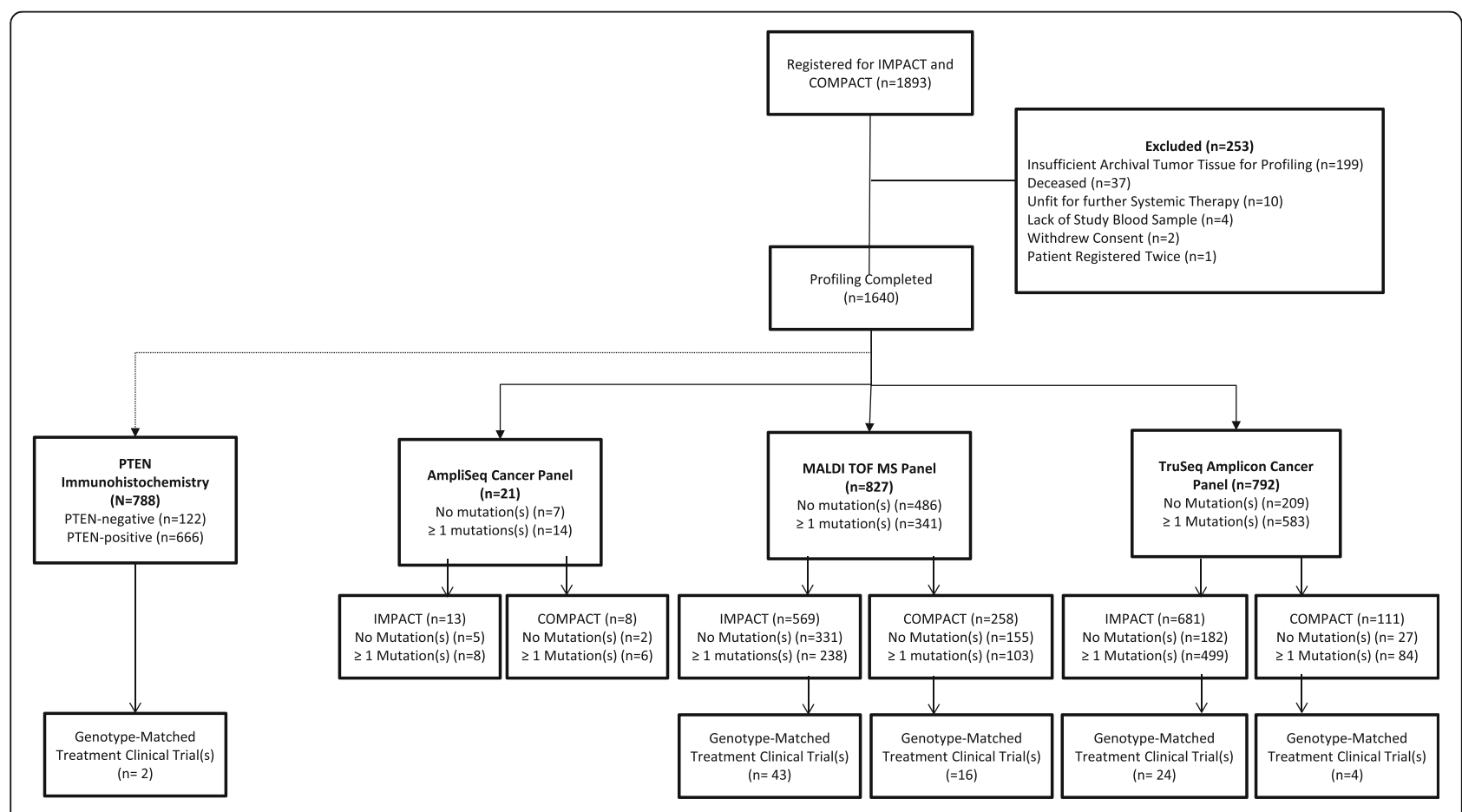

Fig. 1 CONSORT diagram 
variants), followed by $K R A S, P I K 3 C A$, and $A P C$ with mutation frequencies in the range of $5-15 \%$ and the remainder $<5 \%$ (Fig. 2). We attribute the difference in mutation landscape between these two platforms to inclusion of TP53 in the TSACP assay but not in MALDI-TOF (see Additional file 1: Supplemental Methods).

Class 1 and 2 variants are the most clinically significant with known actionability for the specific variant in the tumor site tested (Class 1 ) or a different tumor site (Class 2) [4]. More than $20 \%$ of patients with breast, colorectal, gynecologial, lung, or pancreatobiliary cancers had Class 1 or 2 variants detected by TSACP or MALDI-TOF (Fig. 3). Of patients with genitourinary cancers, only $9 \%$ had actionable variants identified on TSACP and $3 \%$ on MALDI-TOF. For patients with other solid tumors, $25 \%$ had actionable variants identified on TSACP and $18 \%$ on MALDI-TOF. PTEN protein expression was lost by IHC for 122/788 (15 \%) tumors tested. PTEN gene mutations were detected by NGS in $14 / 122(11.5 \%)$ tumors that were PTEN-negative by IHC.

\section{Clinical trials and outcomes}

Of the 1640 patients with molecular profiling results, $245(15 \%)$ were subsequently enrolled in 277 therapeutic clinical trials, including $84(5 \%)$ treated on 89 genotype-matched trials (Table 2). Patients with pancreatobiliary, upper aerodigestive tract, and other solid tumors were least likely to be treated on genotypematched trials. Somatic mutations in four genes (PIK3CA, KRAS, BRAF, and EGFR) accounted for $76 / 89$ (85\%) of genotype-matched trial enrollments: including PIK3CA for breast cancer (20/22); BRAF (5/18) and KRAS (9/18) for colorectal cancer; KRAS (9/18) and EGFR (7/18) for non-small cell lung cancer; and KRAS $(14 / 22)$ and PIK3CA (7/22) for gynecological cancers. A

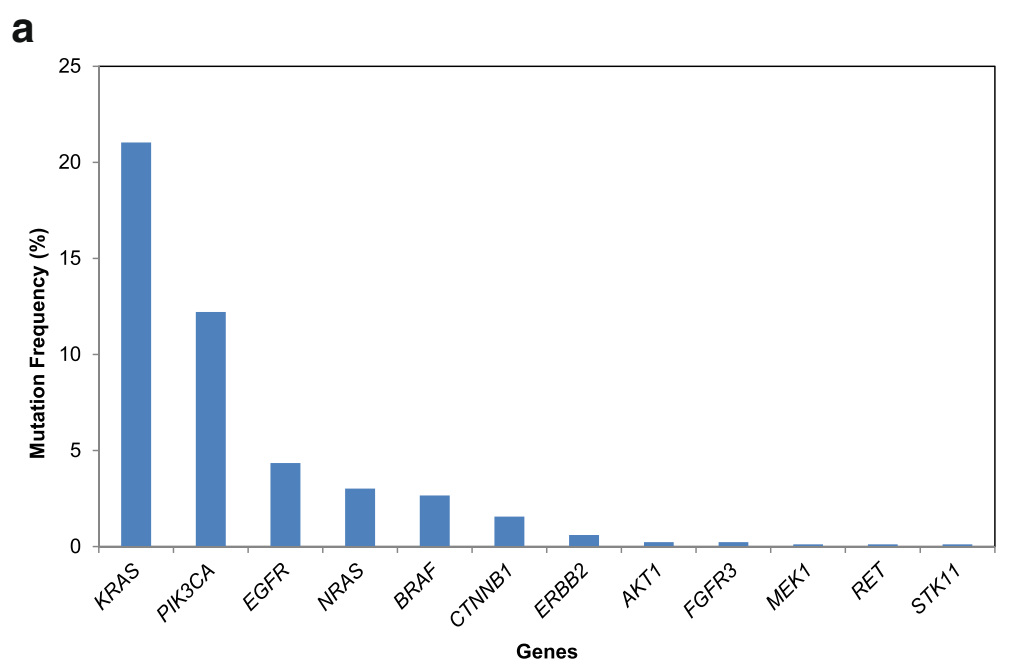

b

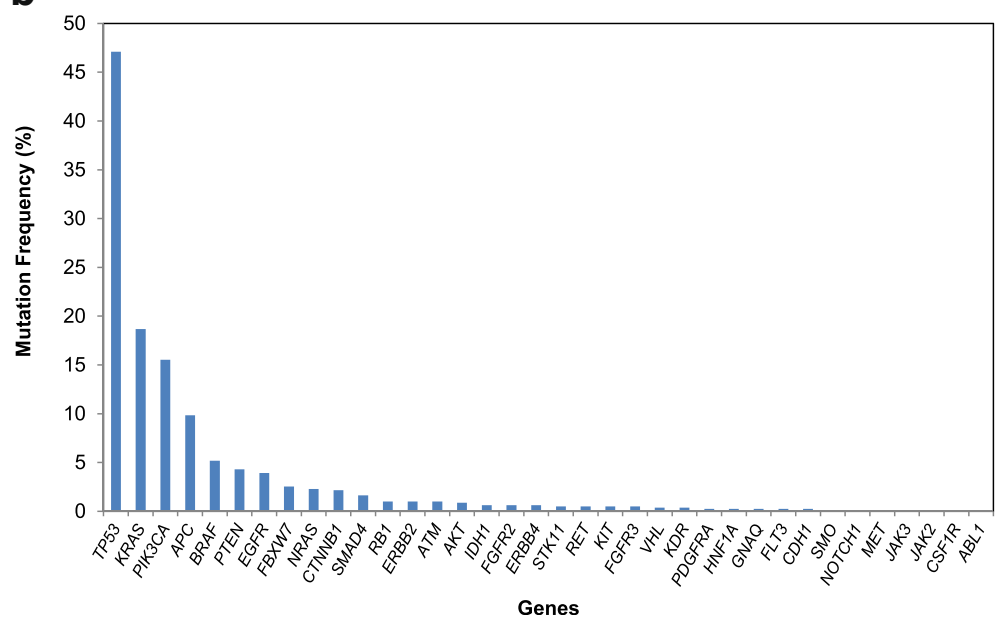

Fig. 2 Mutation frequency by gene from results of (a) MALDI-TOF, $n=827$, and (b) TruSeq Amplicon Cancer Panel, $n=792$. Mutation frequency was calculated as number of variant occurrences within each gene divided by the total number of patients 

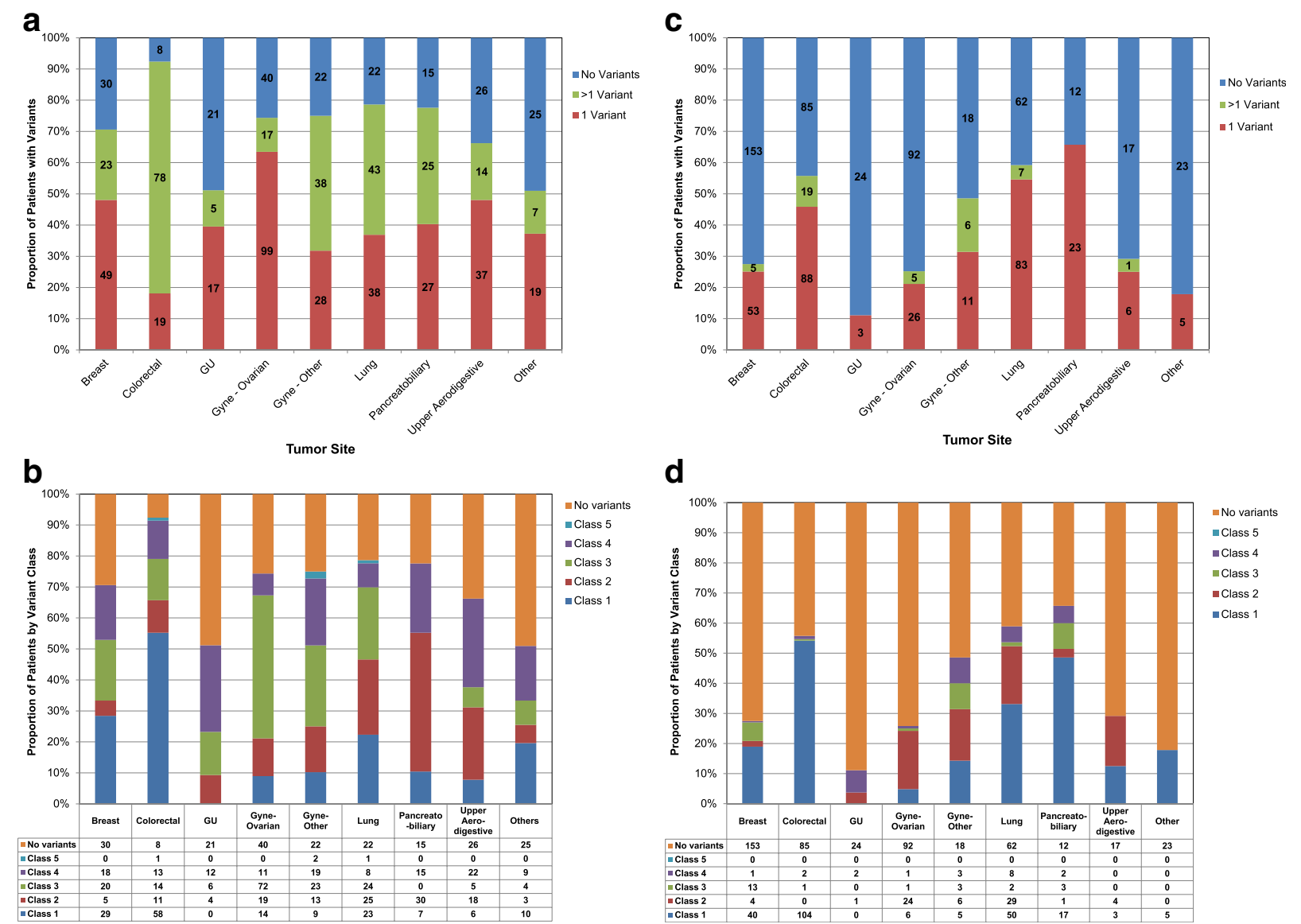

Fig. 3 Distribution of patients by tumor site and most actionable variant identified [4]. Cases tested with TruSeq Amplicon Cancer Panel (TSACP; $n=792)$ are shown in $(\mathbf{a})$ and $(\mathbf{b})$; cases tested by MALDI-TOF MS ( $n=827$ ) are shown in (c) and (d). a Proportion and number of variants by tumor site, TSACP. b Actionability of variants by tumor site, TSACP. c Proportion and number of variants by tumor site, MALDI-TOF. d Actionability of variants per case by tumor site, MALDI-TOF. Patients with more than one variant were counted once by their most actionable variant class. Total number of patients is indicated by value within or below each bar section. "Gyne-other" includes cervical, endometrial, fallopian tube, uterine, and vulvar

complete list of genotype-matched clinical trials by drug class, somatic genotype (variant level), and tumor type are summarized in Table 3.

The age and sex distribution, as well as the number of lines of prior systemic therapy, were similar between the genotype-matched and genotype-unmatched trial patient cohorts (Table 2). There was no difference in the proportion of trials that were genotype-matched between patients profiled on MALDI-TOF MS (61/176 [35 \%]) compared with TSACP $(28 / 101$ [28 \%]; $p=0.24)$. A higher proportion of genotype-matched trial patients were treated in phase I studies $(81 \%)$ compared with genotypeunmatched trials $(46 \% ; p<0.001)$. Genotype-matched trial patients were more likely to be treated with targeted drug combinations without chemotherapy or immunotherapy. The overall response rate was higher in patients treated on genotype-matched trials (19\%) compared with genotype-unmatched trials ( $9 \% ; p=0.026)$ (Fig. 4). In multi-variable analysis, trial matching according to genotype $(p=0.021)$ and female gender $(p=0.034)$ were the only statistically significant factors associated with response (Additional file 1: Table S4). Genotype-matched trial patients were more likely to achieve a best response of any shrinkage in the sum of their target lesions (62\%) compared with genotype-unmatched trial patients (32\%; $p<0.001)$. There was no difference in the time on treatment (15 months versus 15 months; $p=0.12$ ) or overall survival (16 months versus 13 months; $p=0.10$ ) for patients treated on genotype-matched versus genotypeunmatched trials.

\section{Germline testing}

Of the patients who were asked during consent about return of incidental pathogenic germline mutations, 658/ 698 (94.3\%) indicated that they wished to receive these results. Two patients were identified with TP53 variants 
Table 2 Characteristics of patients enrolled in therapeutic trials following molecular profiling

\begin{tabular}{|c|c|c|c|c|}
\hline & All trials & $\begin{array}{l}\text { Genotype- } \\
\text { matched }\end{array}$ & $\begin{array}{l}\text { Genotype- } \\
\text { unmatched }\end{array}$ & $p$ value \\
\hline Median age (range) & $58(18-81)$ & $58(24-81)$ & $58.5(18-80)$ & NS \\
\hline Female/Male & $205 / 72$ & $64 / 25$ & $141 / 47$ & NS \\
\hline $\begin{array}{l}\text { Median prior systemic } \\
\text { therapies (range) }\end{array}$ & $2(1-16)$ & $2(1-11)$ & $2(1-16)$ & NS \\
\hline \multicolumn{5}{|c|}{ Tumor type (number of patients) } \\
\hline Breast & 47 & 22 & 25 & \multirow[t]{5}{*}{ NS } \\
\hline Colorectal & 43 & 18 & 25 & \\
\hline Lung & 48 & 18 & 30 & \\
\hline Gynecological & 91 & 22 & 69 & \\
\hline Other & 48 & 9 & 39 & \\
\hline \multicolumn{5}{|c|}{ Genotyping platform (number of patients) } \\
\hline MALDI-TOF MS panel & 176 & 61 & 115 & \multirow[t]{3}{*}{ NS } \\
\hline $\begin{array}{l}\text { TruSeq Amplicon } \\
\text { cancer panel }\end{array}$ & 101 & 28 & 73 & \\
\hline $\begin{array}{l}\text { Ampliseq cancer } \\
\text { panel }\end{array}$ & 0 & 0 & 0 & \\
\hline \multicolumn{5}{|c|}{ Trial phase (number of patients) } \\
\hline Phase I & 158 & 72 & 86 & \multirow[t]{3}{*}{$<0.001$} \\
\hline Phase II & 67 & 9 & 58 & \\
\hline Phase III & 52 & 8 & 44 & \\
\hline \multicolumn{5}{|c|}{ Investigational agent(s) (number of patients) } \\
\hline $\begin{array}{l}\text { Targeted } \\
\text { monotherapy }\end{array}$ & 112 & 23 & 89 & \multirow[t]{5}{*}{$<0.001$} \\
\hline $\begin{array}{l}\text { Targeted drug } \\
\text { combination }\end{array}$ & 87 & 59 & 28 & \\
\hline $\begin{array}{l}\text { Targeted drug and } \\
\text { chemotherapy }\end{array}$ & 43 & 7 & 36 & \\
\hline Immunotherapy & 34 & 0 & 34 & \\
\hline Radiotherapy & 1 & 0 & 1 & \\
\hline
\end{tabular}

NS not significant

in DNA extracted from blood. The first patient was a 36-year-old woman diagnosed with metastatic breast cancer, with a prior papillary thyroid cancer at the age of 28 years, who had a heterozygous germline TP53 c.817C > T (p.Arg273Cys) pathogenic mutation. Her family history was notable for her mother who died from cancer of unknown primary at the age of 63 years and a maternal aunt with breast cancer at the age of 62 years. The second patient, a 77-year-old woman diagnosed with metastatic cholangiocarcinoma, had no family history of malignancy. We detected a heterozygous TP53 c.524G > A (p.Arg175His) pathogenic mutation at $15 \%$ allele frequency in the blood that was not present in tumor. This finding is not consistent with inherited LiFraumeni syndrome (LFS), but may represent either clonal mosaicism or an age-related or treatment-related mutation limited to blood.

\section{Discussion}

We demonstrated that molecular profiling with massspectrometry-based genotyping or targeted NGS can be implemented in a large academic cancer center to identify patients with advanced solid tumors who are candidates for genotype-matched clinical trials. The rapid enrolment to our study reflects the high level of motivation of patients and their oncologists to pursue genomic testing that has been previously reported by our group [9, 10] and others [1, 11-13]. Disappointingly, only $5 \%$ of patients who underwent successful molecular profiling in our study were subsequently treated on genotype-matched clinical trials, consistent with other centers. For comparison, the MD Anderson institutional genomic testing protocol matched 83/ 2000 (4\%) of patients [1], the SAFIR-01 breast cancer trial matched 28/423 (7 \%) [14], and the British Columbia Cancer Agency Personalized Oncogenomics Trial matched 1/100 (1\%) [15]. To facilitate trial accrual, we incorporated multidisciplinary tumor board discussions, physician-directed email alerts with genotypematched trial listings available at our institution, and individual physician summaries of profiling results. In spite of these efforts, the rate of genotype-matched clinical trial enrolment was low, due to patient deterioration, lack of available clinical trials, and unwillingness of patients to travel for clinical trial participation. There was no difference in proportion of patients treated on genotypematched trials who underwent profiling using MALDITOF or a larger targeted NGS panel. This highlights how few somatic mutations are truly "druggable" through clinical trial matching, even in a large academic cancer center with a broad portfolio of phase I/II trials.

A key finding of our study is that patients in genotypematched trials were more likely to achieve response than patients in genotype-unmatched trials. Albeit a nonrandomized comparison, this finding comprises an important metric and distinguishes our molecular profiling program from other prospective studies that have not tracked longitudinal clinical outcome [1, 16, 17]. Von Hoff and colleagues were the first to report clinical outcome from a prospective molecular profiling (MP) study, with 18/66 (27 \%) of patients who received treatment guided by MP data, including RNA-expression profiling and immunohistochemistry (IHC) or fluorescence in situ hybridization (FISH) testing for 11 markers, achieving a progression-free survival (PFS) ratio on MPselected therapy/PFS on prior therapy) of $\geq 1.3$ [18]. This study was performed prior to the era of multiplex mutation testing and many patients received MP-guided therapy with cytotoxic therapy using biomarker data that has not been shown to influence treatment response. An analysis of 1114 patients treated on investigational clinical trials at the Clinical Center for Targeted Therapy at MD 
Table 3 Genotype-matched clinical trials by drug class, somatic genotypes (variant level), and tumor type $(n=89)$

\begin{tabular}{|c|c|c|c|}
\hline Tumor type & $\begin{array}{l}\text { Somatic genotype } \\
\text { (variant) }\end{array}$ & $\begin{array}{l}\text { Genotype-matching } \\
\text { trial drug class }\end{array}$ & $\begin{array}{l}\text { Target lesion } \\
\text { percent change }\end{array}$ \\
\hline Colorectal & $\begin{array}{l}\text { No mutations } \\
\text { (KRAS wildtype) }\end{array}$ & EGFR & $-100 \%$ \\
\hline Breast & PIK3CA E545K & PI3K, Endocrine & $-90 \%$ \\
\hline Colorectal & BRAF V600E & BRAF, PI3K, EGFR & $-85 \%$ \\
\hline \multirow[t]{2}{*}{ Colorectal } & BRAF V600E & BRAF, PI3K, EGFR & $-81 \%$ \\
\hline & TP53 S215G & & \\
\hline Gynecological & KRAS G13D & PI3K, MEK & $-70 \%$ \\
\hline \multirow[t]{2}{*}{ Gynecological } & PIK3CA H1047R & VEGF & $-64 \%$ \\
\hline & KRAS G12D & & \\
\hline Gynecological & KRAS G12V & PI3K, MEK & $-63 \%$ \\
\hline Breast & PIK3CA H1047R & PI3K, IGF1R & $-61 \%$ \\
\hline \multirow[t]{2}{*}{ Lung } & EGFR E746_A750del & EGFR & $-58 \%$ \\
\hline & EGFR T790M & & \\
\hline Lung & KRAS G13D & MEK & $-54 \%$ \\
\hline Breast & PIK3CA H1047R & PI3K & $-50 \%$ \\
\hline \multirow[t]{3}{*}{ Lung } & EGFR L858R & EGFR & $-47 \%$ \\
\hline & EGFR T790M & & \\
\hline & CTNNB1 S37C & & \\
\hline Gynecological & KRAS G12D & MEK & $-47 \%$ \\
\hline \multirow[t]{2}{*}{ Gynecological } & KRAS G12V & PI3K, IGF1R & $-45 \%$ \\
\hline & NRAS Q61R & & \\
\hline Gynecological & KRAS G12A & PI3K, MEK & $-38 \%$ \\
\hline Breast & PIK3CA N345K & ANG2, MTOR & $-37 \%$ \\
\hline Lung & KRAS G12D & PI3K, MEK & $-37 \%$ \\
\hline \multirow[t]{2}{*}{ Gynecological } & PIK3CA H1047R & PI3K, MEK & $-37 \%$ \\
\hline & KRAS G12D & & \\
\hline Lung & KRAS G12C & MEK & $-28 \%$ \\
\hline Gynecological & TP53 K132N & WEE1 & $-26 \%$ \\
\hline Gynecological & KRAS G12D & PI3K, MEK & $-25 \%$ \\
\hline \multirow[t]{4}{*}{ Colorectal } & BRAF V600E & BRAF, PI3K, EGFR & $-24 \%$ \\
\hline & TP53 R273C & & \\
\hline & PIK3CA Q546K & & \\
\hline & APC E1544X & & \\
\hline \multirow[t]{2}{*}{ Breast } & ERBB2 D769H & HER2 & $-23 \%$ \\
\hline & PIK3CA N345K & & \\
\hline $\begin{array}{l}\text { Upper } \\
\text { aerodigestive }\end{array}$ & BRAF V600E & MEK & $-20 \%$ \\
\hline Lung & KRAS G12S & MEK & $-20 \%$ \\
\hline Gynecological & KRAS G12D & MEK, PI3K & $-20 \%$ \\
\hline \multirow[t]{3}{*}{ Colorectal } & BRAF VGO0E & BRAF, PI3K, EGFR & $-20 \%$ \\
\hline & TP53 R175H & & \\
\hline & TP53 Q165X & & \\
\hline Breast & FGFR2 Y376C & FGFR & $-19 \%$ \\
\hline Breast & PIK3CA H1047L & PI3K & $-18 \%$ \\
\hline
\end{tabular}

Table 3 Genotype-matched clinical trials by drug class, somatic genotypes (variant level), and tumor type $(n=89)$ (Continued)

\begin{tabular}{|c|c|c|c|}
\hline Other & GNAQ Q209P & MEK & $-18 \%$ \\
\hline Lung & EGFR L858R & HER3, EGFR & $-18 \%$ \\
\hline Lung & KRAS G12A & PI3K, MEK & $-17 \%$ \\
\hline Lung & EGFR L858R & HER3, EGFR & $-17 \%$ \\
\hline \multirow[t]{2}{*}{ Colorectal } & KRAS G12D & PI3K, MEK & $-16 \%$ \\
\hline & PIK3CA E545K & & \\
\hline Gynecological & KRAS G12V & PI3K, MEK & $-16 \%$ \\
\hline Gynecological & BRAF VGO0E & MEK & $-15 \%$ \\
\hline Gynecological & KRAS G12D & PI3K, MEK & $-15 \%$ \\
\hline Lung & KRAS G12V & PI3K, MEK & $-13 \%$ \\
\hline Colorectal & KRAS G12S & MEK, EGFR & $-13 \%$ \\
\hline Gynecological & NRAS Q61K & PI3K, MEK & $-13 \%$ \\
\hline Pancreatobiliary & KRAS G12V & PI3K, IGF1R & $-13 \%$ \\
\hline Gynecological & PIK3CA H1047R & ANG2, mTOR & $-9 \%$ \\
\hline Breast & PIK3CA E545K & AKT & $-7 \%$ \\
\hline Colorectal & KRAS G12V & MEK, EGFR & $-7 \%$ \\
\hline \multirow[t]{2}{*}{ Breast } & ERBB2 D769H & PI3K, IGF1R & $-7 \%$ \\
\hline & PIK3CA N345K & & \\
\hline Lung & KRAS G12V & MEK & $-6 \%$ \\
\hline \multirow[t]{4}{*}{ Colorectal } & ERBB2 L755S & VEGF, ANG2 & $-6 \%$ \\
\hline & BRAF N581S & & \\
\hline & ERBB2 L755S & & \\
\hline & APC Q1429fs & & \\
\hline \multirow[t]{2}{*}{ Genitourinary } & No mutations & PI3K & $-5 \%$ \\
\hline & $\begin{array}{l}\text { PTEN negative } \\
\text { on IHC }\end{array}$ & & \\
\hline Lung & EGFR L858R & EGFR & $-4 \%$ \\
\hline Lung & EGFR L858R & EGFR & $-4 \%$ \\
\hline Lung & EGFR E746_A750del & EGFR & $-3 \%$ \\
\hline Breast & PIK3CA H1047R & AKT & $-3 \%$ \\
\hline $\begin{array}{l}\text { Upper } \\
\text { aerodigestive }\end{array}$ & KRAS G12V & PI3K, MEK & $-2 \%$ \\
\hline Gynecological & KRAS G12D & PI3K, MEK & $-2 \%$ \\
\hline Colorectal & KRAS G13D & MEK, EGFR & $-0.6 \%$ \\
\hline \multirow[t]{2}{*}{ Genitourinary } & No mutations & PI3K & $0 \%$ \\
\hline & $\begin{array}{l}\text { PTEN negative } \\
\text { on IHC }\end{array}$ & & \\
\hline Breast & PIK3CA H1047R & PI3K & $0 \%$ \\
\hline \multirow[t]{2}{*}{ Colorectal } & PIK3CA E542K & PI3K & $+0.5 \%$ \\
\hline & KRAS G12V & & \\
\hline Colorectal & $\begin{array}{l}\text { No Mutations } \\
\text { (KRAS wildtype) }\end{array}$ & MEK, EGFR & $+2 \%$ \\
\hline \multirow[t]{2}{*}{ Colorectal } & KRAS G13D & PI3K, MEK & $+2 \%$ \\
\hline & PIK3CA E545K & & \\
\hline Breast & PIK3CA H1047L & PI3K, IGF1R & $+4 \%$ \\
\hline Genitourinary & No mutations & PI3K & $+4 \%$ \\
\hline
\end{tabular}


Table 3 Genotype-matched clinical trials by drug class, somatic genotypes (variant level), and tumor type $(n=89)$ (Continued)

\begin{tabular}{|c|c|c|c|}
\hline & $\begin{array}{l}\text { PTEN negative } \\
\text { on IHC }\end{array}$ & & \\
\hline Gynecological & PIK3CA E545K & MTOR & $+5 \%$ \\
\hline Breast & PIK3CA N345K & PI3K, IGF1R & $+6 \%$ \\
\hline \multirow[t]{2}{*}{ Breast } & PIK3CA N345K & PI3K, MEK & $+6 \%$ \\
\hline & NRAS G12D & & \\
\hline Gynecological & PIK3CA C42OR & FGFR, PI3K & $+8 \%$ \\
\hline Colorectal & KRAS G12D & MEK, EGFR & +9\% \\
\hline Lung & PIK3CA E545K & PI3K, IGF1R & $+11 \%$ \\
\hline Colorectal & $\begin{array}{l}\text { No mutations } \\
\text { (KRAS wildtype) }\end{array}$ & MEK, EGFR & $+11 \%$ \\
\hline \multirow[t]{2}{*}{ Colorectal } & KRAS G12D & PI3K, MEK & $+12 \%$ \\
\hline & PIK3CA Q546K & & \\
\hline Gynecological & PIK3CA H1047R & PI3K, IGF1R & $+12 \%$ \\
\hline \multirow[t]{3}{*}{ Breast } & PIK3CA p.Glu545Gly & AKT & $+28 \%$ \\
\hline & PTEN p.Leu320X & & \\
\hline & PIK3CA p.Arg93Gln & & \\
\hline \multirow[t]{3}{*}{ Gynecological } & TP53 R175H & PI3K & $+29 \%$ \\
\hline & PIK3CA R93W & & \\
\hline & FBXW7 R479Q & & \\
\hline \multirow[t]{2}{*}{ Colorectal } & KRAS G12D & PI3K, MEK & $+30 \%$ \\
\hline & PIK3CA E545K & & \\
\hline Genitourinary & PIK3CA p.Asn345Lys & PI3K & $+31 \%$ \\
\hline Breast & PIK3CA E545K & FGFR, PI3K & $+32 \%$ \\
\hline Breast & PIK3CA H1047R & PI3K, IGF1R & $+39 \%$ \\
\hline Colorectal & $\begin{array}{l}\text { No mutations } \\
\text { (KRAS wildtype) }\end{array}$ & MEK, EGFR & $+55 \%$ \\
\hline Breast & PIK3CA E542K & PI3K, Endocrine & $+66 \%$ \\
\hline Breast & PIK3CA N345K & PI3K, IGF1R & $\mathrm{NE}$ \\
\hline Lung & KRAS G12C & PI3K, MEK & $\mathrm{NE}$ \\
\hline Breast & PIK3CA H1047R & PI3K, IGF1R & $\mathrm{NE}$ \\
\hline \multirow[t]{2}{*}{ Lung } & KRAS G12A & MEK & $\mathrm{NE}$ \\
\hline & PIK3CA H1047R & & \\
\hline Gynecological & KRAS G12A & PI3K, IGF1R & NE \\
\hline \multirow[t]{3}{*}{ Breast } & PIK3CA E545K & PI3K & NE \\
\hline & TP53 L252del & & \\
\hline & BRAF C.1315-4C > G & & \\
\hline Gynecological & KRAS G12V & MEK & $\mathrm{NE}$ \\
\hline Lung & BRAF V600E & MEK & $\mathrm{NE}$ \\
\hline \multirow[t]{2}{*}{ Breast } & PIK3CA H1047L & PI3K & $\mathrm{NE}$ \\
\hline & TP53 C238Y & & \\
\hline Other & KIT V559A & PI3K, EGFR & NE \\
\hline
\end{tabular}



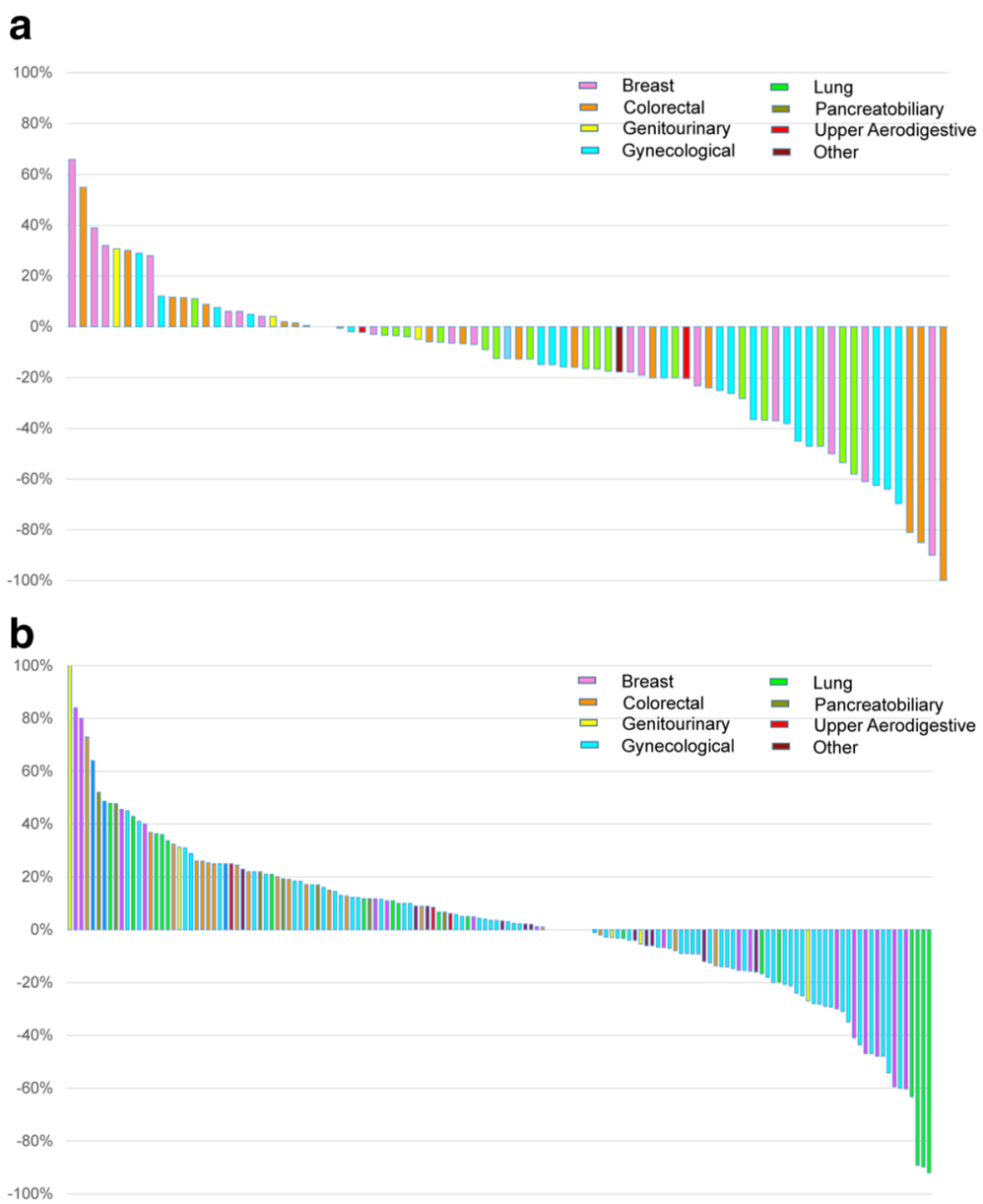

Fig. 4 a Waterfall plot of best tumor shrinkage of target lesions by RECIST for patients treated on (a) genotype-matched clinical trials $(n=79)$ and (b) genotype-unmatched clinical trials $(n=150)$

therapy based on ERBB2 (HER2) amplification. Since ER and HER2 testing are routinely performed in breast cancer patients to guide standard therapies, these patients would not have been included in our matched therapy cohort if the ER and HER2 status were known prior to enrollment in our molecular profiling study.

The only randomized trial that has prospectively assessed the utility of molecular profiling (SHIVA) reported no difference in objective response or PFS for patients treated with genotype-matched versus standard treatments [13]. More than $40 \%$ of patients randomized in the SHIVA trial did not have genomic alterations identified and were included based upon expression of hormone receptors. Patients were matched to a limited range of approved targeted agents following a predefined algorithm that did not include best-in-class investigational agents that are being tested in early phase clinical trials. Despite the negative results of SHIVA, enthusiasm to conduct genomic-based clinical trials such as NCIMATCH [12] [NCT02465060], and LUNG-MAP [22] [NCT02154490] remains strong to further define the value of precision medicine. The findings of our study, in which the majority of patients treated on genotype-matched trials were enrolled in phase I targeted therapy trials, are consistent with a recent meta-analysis of phase I trials that demonstrated a higher overall response rate $(30.6 \%$ versus $4.9 \%$, $p<0.001)$ and median PFS $(5.7$ months versus 2.95 months, $p<0.001$ ) for targeted therapy trials that used biomarker-selection compared with those that did not [23].

Measuring the clinical utility of molecular profiling is difficult [3]. We did not comprehensively capture how testing results influenced clinical decisions outside of therapeutic clinical trial enrolment, such as reclassification of tumor subtype and site of primary based on mutation results. For example, we enrolled a patient 
with an unknown primary cancer with intra-abdominal metastases that was found to harbor a somatic IDH1 p.Arg132Cys variant, leading to the reclassification as a likely intrahepatic cholangiocarcinoma. We also did not fully evaluate the use of testing results to avoid ineffective standard treatments (i.e. KRAS exon 4 somatic variants in colorectal cancer to inform decision not to use EGFR monoclonal antibody treatment) and treatment with approved targeted agents outside of their approved indications. Few patients in our study received targeted treatments based upon profiling results outside of clinical trials, due to limited access to targeted drugs outside of publicly funded standard-of-care indications in Ontario.

New technological advances are being studied in molecular profiling programs-including larger gene panels [2, 17]; whole exome [16], whole genome (WGS) or RNA sequencing (RNA-Seq) [24, 25]; and integrative systems biology analyses of deregulated cellular pathways [26]. Greater access to clinical trials for genomically characterized patients, such as umbrella and basket trial designs [27], may also improve the success of genotype-treatment matching. To assess whether decision support tools integrated at the point of care can improve enrollment of patients on genotype-matched trials, we are piloting a smart phone application to help physicians identify genotypematched trials for their patients with profiling data.

There are several limitations of our study. Only a single archival sample was profiled for each patient, often obtained many years prior to molecular testing. Fresh biopsy of a current metastatic lesion for molecular profiling at the time of study enrolment may have yielded different results due to clonal evolution or tumor heterogeneity [28]. Our genomic testing was limited to hotspot point mutation testing or limited targeted sequencing and did not include gene copy number alterations or recurrent translocations that may be important for the selection of genotype-matched therapy. There were patients identified with potentially "druggable" mutations who were candidates for genotype-matched trials; however, they could not be enrolled because of the constraints of slot allocation in early phase clinical trials across multiple institutions or were deemed ineligible due to trial-specific exclusion criteria. Our study population also included many patients with heavily pre-treated metastatic disease who were not well enough for further therapy when results of molecular testing were reported. In addition, tumor response is an imperfect surrogate endpoint to assess therapeutic benefit in early phase clinical trials that should interpreted with caution [28]. We did not observe a difference in time on treatment or overall survival for patients treated on genotype-matched versus genotype-unmatched clinical trials. PFS data were not available in our cohort precluding a comparison of the outcome of genotype-matched therapy with the immediate prior line of treatment, as has been reported by other investigators [13, 14, 21].

\section{Conclusions}

We provide preliminary evidence that genotype-matched trial treatment selected on the basis of molecular profiling was associated with increased tumor shrinkage, although only a small proportion of profiled patients benefitted from this approach. Through this initiative, we have created a valuable repository of data and tumor samples that are amenable to additional research and data sharing initiatives. Greater efforts should be made to expand opportunities for genotype-trial matching and further studies are needed to evaluate the clinical utility of targeted NGS profiling.

\section{Additional file}

Additional file 1: Supplementary methods and Tables S1-S4 (DOCX $148 \mathrm{~kb}$ )

\section{Acknowledgements}

The authors acknowledge Swati Garg, PhD, and Mariam Thomas, PhD, Princess Margaret Cancer Centre, for their contributions to variant data analysis. They are also thankful to the all of the medical oncologists, pathologists, laboratory technicians, clinical data coordinators, and correlative studies coordinators who participated in this research study.

\section{Funding}

This work was supported by the Princess Margaret Cancer Foundation; the Cancer Care Ontario Applied Clinical Research Unit [to LLS]; the University of Toronto Division of Medical Oncology Strategic Innovation [to PLB]; and the Ontario Ministry of Health and Long-Term Care Academic Health Sciences Centre Alternate Funding Plan Innovation Award [to PLB].

\section{Availability of data and materials}

The dataset supporting the results of this article is available in the American Association for Cancer Research Project Genomics, Evidence, Neoplasia, Information, Exchange [GENIE] (http://www.aacr.org/Research/Research/ Pages/aacr-project-genie.aspx\#.WAWFw2XqeDo).

\section{Authors' contributions}

TLS and PLB had full access to all of the data in the study and take responsibility for the integrity of the data and accuracy of the data analysis. LLS, PLB, SK-R, and $\mathrm{CY}$ conceived of the study concept and wrote the protocol. All authors participated in the acquisition, analysis, or interpretation of data. TS, SK-R, LLS, CY, and PLB drafted the manuscript for initial review by all authors. LW performed statistical analysis. All authors read and approved the final manuscript.

\section{Authors' information}

Not applicable.

\section{Competing interests}

The authors declare that they have no competing interests.

\section{Consent for publication}

Not applicable.

Ethics approval and consent to participate

The University Health Network Research Ethics Board approved this study (\#11-0962-CE). 


\section{Author details}

'Laboratory Medicine Program, University Health Network, Toronto, Canada. ${ }^{2}$ Department of Laboratory Medicine and Pathobiology, University of Toronto, Toronto, Canada. ${ }^{3}$ Cancer Genomics Program, Princess Margaret Cancer Centre, Toronto, Canada. ${ }^{4}$ Division of Medical Oncology and Hematology, Princess Margaret Cancer Centre, 610 University Avenue, Toronto M5G 2M9, Canada. ${ }^{5}$ Department of Medicine, University of Toronto, Toronto, Canada. ${ }^{6}$ Department of Medical Biophysics, University of Toronto, Toronto, Canada. 'Department of Oncology, Grand River Regional Cancer Centre, Kitchener-Waterloo, Canada. ${ }^{8}$ Department of Oncology, McMaster University, Faculty of Health Sciences, Hamilton, Canada. ${ }^{9}$ Department of Medicine, Markham Stouffville Hospital, Markham, Canada. ${ }^{10}$ Department of Biostatistics, Princess Margaret Cancer Centre, Toronto, Canada. ${ }^{11}$ Princess Margaret Research Institute, Princess Margaret Cancer Centre, Toronto, Canada.

\section{Received: 19 June 2016 Accepted: 11 October 2016}

\section{Published online: 25 October 2016}

\section{References}

1. Meric-Bernstam F, Brusco L, Shaw K, Horombe C, Kopetz S, Davies MA, et al. Feasibility of large-scale genomic testing to facilitate enrollment onto genomically matched clinical trials. J Clin Oncol. 2015;33:2753-62.

2. Lacombe D, Tejpar S, Salgado R, Cardoso F, Golfinopoulos V, Aust D, et al. European perspective for effective cancer drug development. Nat Rev Clin Oncol. 2014:11(8):492-8.

3. Hyman DM, Solit DB. Tumor genetic screening programs: a call to action. J Clin Oncol. 2015;33(25):2725-6.

4. Sukhai MA, Craddock KJ, Thomas M, Hansen AR, Zhang T, Siu L, et al. A classification system for clinical relevance of somatic variants identified in molecular profiling of cancer. Genet Med. 2016;18:128-36.

5. Yanagawa N, Leduc C, Kohler D, Saieg MA, John T, Sykes J, et al. Loss of phosphatase and tensin homolog protein expression is an independent poor prognostic marker in lung adenocarcinoma. J Thorac Oncol. 2012;7(10):1513-21.

6. Eisenhauer EA, Therasse P, Bogaerts J, Schwartz LH, Sargent D, Ford R, et al. New response evaluation criteria in solid tumours: revised RECIST guideline (version 1.1). Eur J Cancer. 2009:45(2):228-47.

7. Touloumis A, Agresti A, Kateri M. GEE for multinomial responses using a local odds ratios parameterization. Biometrics. 2013;69(3):633-40.

8. Borgan, Ø. (2001), Modeling Survival Data: Extending the Cox Model. Terry M. Therneau and Patricia M. Grambsch, Springer-Verlag, New York, 2000. doi:10.1002/sim.956.

9. Miller FA, Hayeems RZ, Bytautas JP, Bedard PL, Ernst S, Hirte H, et al. Testing personalized medicine: patient and physician expectations of next-generation genomic sequencing in late-stage cancer care. Eur J Hum Genet. 2014:22(3):391-5.

10. Blanchette PS, Spreafico A, Miller FA, Chan K, Bytautas J, Kang S, et al. Genomic testing in cancer: Patient knowledge, attitudes, and expectations. Cancer. 2014;120(19):3066-73.

11. André F, Bachelot T, Commo F, Campone M, Arnedos M, Dieras V, et al. Comparative genomic hybridisation array and DNA sequencing to direct treatment of metastatic breast cancer: a multicentre, prospective trial (SAFIR01/UNICANCER). Lancet Oncol. 2014;15(3):267-74.

12. Conley BA, Doroshow JH. Molecular analysis for therapy choice: NCI MATCH. In: Seminars in oncology: 2014. Elsevier; 2014: p. 297-299.

13. Le Tourneau C, Delord J-P, Gonçalves A, Gavoille C, Dubot C, Isambert N et al. Molecularly targeted therapy based on tumour molecular profiling versus conventional therapy for advanced cancer (SHIVA): a multicentre, open-label, proof-of-concept, randomised, controlled phase 2 trial. Lancet Oncol. 2015;16:1324-34.

14. Von Hoff DD, Stephenson JJ, Rosen P, Loesch DM, Borad MJ, Anthony S, et al. Pilot study using molecular profiling of patients' tumors to find potential targets and select treatments for their refractory cancers. J Clin Oncol. 2010;28(33):4877-83.

15. Laskin J, Jones S, Aparicio S, Chia S, Ch'ng C, Deyell R, et al. Lessons learned from the application of whole-genome analysis to the treatment of patients with advanced cancers. Mol Case Stud. 2015;1(1):a000570.

16. Van Allen EM, Wagle N, Stojanov P, Perrin DL, Cibulskis K, Marlow S, et al. Whole-exome sequencing and clinical interpretation of formalin-fixed, paraffin-embedded tumor samples to guide precision cancer medicine. Nat Med. 2014;20(6):682-8
17. Cheng DT, Mitchell TN, Zehir A, Shah RH, Benayed R, Syed A, et al. Memorial Sloan Kettering-Integrated Mutation Profiling of Actionable Cancer Targets (MSK-IMPACT): a hybridization capture-based nextgeneration sequencing clinical assay for solid tumor molecular oncology. J Mol Diagn. 2015;17(3):251-64.

18. Von Hoff D, Stephenson Jr J, Rosen P, Loesch D, Borad M, Anthony S, et al. Pilot study using molecular profiling of patients' tumors to find potential targets and select treatments for their refractory cancers. J Clin Oncol Off J Am Soc Clin Oncol. 2010;28(33):4877.

19. Tsimberidou A-M, Iskander NG, Hong DS, Wheler JJ, Falchook GS, Fu S, et al. Personalized medicine in a phase I clinical trials program: the MD Anderson Cancer Center initiative. Clin Cancer Res. 2012;18(22):6373-83.

20. Wheler JJ, Janku F, Naing A, Li Y, Stephen B, Zinner RG, et al. Cancer therapy directed by comprehensive genomic profiling: a single center study. Cancer Res. 2016:76:3690-701.

21. Schwaederle M, Parker BA, Schwab RB, Daniels GA, Piccioni DE, Kesari S, et al. Precision Oncology: The UC San Diego Moores Cancer Center PREDICT Experience. Mol Cancer Ther. 2016;15(4):743-52.

22. Herbst RS, Gandara DR, Hirsch FR, Redman MW, LeBlanc M, Mack PC, et al. Lung Master Protocol (Lung-MAP)—a biomarker-driven protocol for accelerating development of therapies for squamous cell lung cancer: SWOG S1400. Clin Cancer Res. 2015:21(7):1514-24.

23. Schwaederle M, Zhao M, Lee JJ, Lazar V, Leyland-Jones B, Schilsky RL, et al. Association of biomarker-based treatment strategies with response rates and progression-free survival in refractory malignant neoplasms: a metaanalysis. JAMA Oncol. 2016. DOI: 10.1001/jamaoncol.2016.2129.

24. Mody RJ, Wu Y-M, Lonigro RJ, Cao X, Roychowdhury S, Vats P, et al. Integrative clinical sequencing in the management of refractory or relapsed cancer in youth. JAMA. 2015;314(9):913-25.

25. Roychowdhury S, lyer MK, Robinson DR, Lonigro RJ, Wu Y-M, Cao X, et al. Personalized oncology through integrative high-throughput sequencing: a pilot study. Sci Transl Med. 2011;3(111):111ra121.

26. Rodon J, Soria J, Berger R, Batist G, Tsimberidou A, Bresson C, et al. Challenges in initiating and conducting personalized cancer therapy trials: perspectives from WINTHER, a Worldwide Innovative Network (WIN) Consortium trial. Ann Oncol. 2015;26:1791-8.

27. Sleijfer S, Bogaerts J, Siu LL. Designing transformative clinical trials in the cancer genome era. J Clin Oncol. 2013;31(15):1834-41.

28. Gerlinger M, Rowan AJ, Horswell S, Larkin J, Endesfelder D, Gronroos E, et al. Intratumor heterogeneity and branched evolution revealed by multiregion sequencing. N Engl J Med. 2012;366(10):883-92.

\section{Submit your next manuscript to BioMed Central and we will help you at every step:}

- We accept pre-submission inquiries

- Our selector tool helps you to find the most relevant journal

- We provide round the clock customer support

- Convenient online submission

- Thorough peer review

- Inclusion in PubMed and all major indexing services

- Maximum visibility for your research

Submit your manuscript at www.biomedcentral.com/submit
) Biomed Central 